\title{
Can the dark matter annihilation signal be significantly boosted by substructures?
}

\author{
A. N. Baushev \\ DESY, 15738 Zeuthen, Germany \\ Institut für Physik und Astronomie, \\ Universität Potsdam, 14476 Potsdam-Golm, Germany \\ Departamento de Astronomía, \\ Universidad de Chile, Casilla 36-D, \\ Correo Central, Santiago, Chile
}

(Dated: December 25, 2015)

\begin{abstract}
A very general cosmological consideration suggests that, along with galactic dark matter halos, much smaller dark matter structures may exist. These structures are usually called 'clumps', and their mass extends to $10^{-6} M_{\odot}$ or even lower. The clumps should give the main contribution into the signal of dark matter annihilation, provided that they have survived until the present time. Recent observations favor a cored profile for low-mass astrophysical halos. We consider cored clumps and show that they are significantly less firm than the standard NFW ones. In contrast to the standard scenario, the cored clumps should have been completely destroyed inside $\sim 20 \mathrm{kpc}$ from the Milky Way center. The dwarf spheroidals should not contain any dark matter clumps. On the other hand, even under the most pessimistic assumption about the clump structure, the clumps should have survived in the Milky Way at a distance exceeding $50 \mathrm{kpc}$ from the center, as well as in low-density cosmic structures. There they significantly boost the dark matter annihilation. We show that at least $70 \%$ of the clumps endured the primordial structure formation should still exist untouched in the present-day Universe.
\end{abstract}

PACS numbers: 95.35.+d; 95.30.Cq; 98.52.Wz

\section{INTRODUCTION}

A very general cosmological consideration suggests that, along with large dark matter haloes, corresponding to visible astronomical objects, like galaxies or galaxy clusters, much smaller dark matter structures may exist. Indeed, the inflation inevitably leads to a Zel'dovichHarrison-like spectrum of the primordial cosmological perturbations, which is flat and contains perturbations of any scale with approximately the same amplitude [1]. The perturbations with masses lower than some cutoff value $M_{c u t}$ may be destroyed in the early Universe by the free streaming. If the dark matter (hereafter DM) is cold and non-interacting, which is the most popular hypothesis now, $M_{\text {cut }}$ is mainly determined by the mass of dark matter particle $m_{d m}$. Roughly speaking, $M_{\text {cut }} \sim M_{P l}^{3} / m_{d m}^{2}$, where $M_{P l}$ is the Planck mass. For $m_{d m}=100 \mathrm{GeV}$, different estimations of $M_{c u t}$ vary from $10^{-12} M_{\odot}[2]$ to $10^{-6} M_{\odot}[3]$. We denote the clump virial mass and radius by $m_{c l}, r_{c l}$.

We consider the DM particles (hereafter DMPs) of mass $100 \mathrm{GeV}$ not by accident. If we suppose that dark matter was in thermodynamical equilibrium in the early Universe, current DM abundance allows estimating of the DM annihilation cross section $\langle\sigma v\rangle_{a} \simeq 3 \cdot 10^{-26} \mathrm{~cm}^{3} / \mathrm{s}$. This is a typical value for weak interactions, which implies that DMPs may interact weakly. Of course, other explanations of the dark matter abundance are possible, and the 'correct' cross section value can be just a coincidence. However, the weakly-interacting massive particles (WIMPs) are one of the most popular dark matter candidate now. The WIMP mass in realistic models typically exceeds $100 \mathrm{GeV}$. A lighter WIMP is possible, but a theorist should take special precautions to avoid tensions with the LHC results, since a light WIMP is usually accompanied by respectively light charged particles in theories like supersymmetry. Thus the existence of tiny dark matter halos (traditionally named 'clumps') is not a theoretical assumption, but an inevitable property of many models of the cold dark matter, if DMP mass exceeds $\sim 1 \mathrm{MeV}$.

The correct estimation of the dark matter clumpiness is of critical importance, especially for the indirect dark matter detection. Indeed, the number of annihilations in a volume $d V$ in an interval of time $d t$ is[32]

$$
\frac{1}{2}\langle\sigma v\rangle \frac{\rho^{2}}{m_{\chi}^{2}} d \tau d V
$$

where $\langle\sigma v\rangle$ is the averaged multiplication of the DM annihilation cross-section on the relative velocity and $m_{\chi}$ is the DMP mass. Thus the annihilation signal is proportional to the so-called boost factor $C=\left\langle\rho^{2}\right\rangle /\langle\rho\rangle^{2}$, which takes into account the enhancement of the annihilation signal by dark-matter substructures. In the standard cosmological scenarios the smallest halos collapse the first, and therefore have the highest density contrast, making the main contribution to the boost. We can define a characteristic radius $r_{s}$ of each halo where its density profile gets steeper than $r^{-2}$, i.e., $d \log \rho / d \log r=-2$ at $r_{s}$. Then we may introduce the halo concentration $c_{v i r} \equiv r_{s} / R_{v i r}$. In the case of NFW halo, this definition coincides with the standard one.

The information about the smallest DM structures is poor at recent. First of all, astronomical observations of the clumps are impossible, since their gravitational 
potential is too small to hold baryon matter. The theoretical view on the clump structure and velocity distribution of the DM particles is also rather uncertain. The clump mass is very small $\left(<10^{-6} M_{\odot}\right.$ for neutralino dark matter) comparing to the galaxy masses, and results and relationships obtained in standard N-body simulations should be extrapolated on many orders of magnitude [4]. Moreover, as we will see below, the present-day number of clumps strongly depends on their interaction with various baryon objects, like stars, galaxy disc etc. It makes a reliable simulation of clump abundance very difficult.

The main fraction of the clumps formed in the early Universe has not survived to our time. There are many mechanisms of clump demolition, and they can all be divided into two groups. A significant part of clumps was tidally destroyed almost immediately after their occurrence by larger halos during the hierarchical structure formation. We will imply the standard $\Lambda \mathrm{CDM}$ model hereafter in this paper. In the framework of this scenario, the hierarchical clump destruction is not sensitive to the clump density profile. Indeed, the spectrum of primordial perturbations is flat in this case, and objects of different masses collapse almost simultaneously. The time interval between the occurrence of the smallest clumps and more massive objects that capture the clumps and destroy them tidally is smaller than the clump virilization time. Consequently, even if present-day clumps have cusps, they were formed later. Therefore, we may use a simplified estimation of the hierarchical clump destruction by calculating an energy gain per each tidal interaction and the number of tidal interactions [5]: a clump is considered to be destroyed if its internal energy increase due to the tidal shocks exceeds its total energy $|E| \sim G M / R$. This estimation leads to the $\propto d M / M^{2}$ mass spectrum of the clumps survived the structure formation and suggests that only about $0.1-0.5 \%$ of clumps endure the hierarchical tides in the each logarithmic mass interval. N-body simulations [6] support the mass spectrum, but suggest a several times higher clump survival ratio. However, even $0.1-0.5 \%$ of clumps endured are sufficient to provide a very significant boosting [7].

The second large group of clump destruction mechanisms can be named astrophysical. The clumps can be disrupted by separate stars, galaxy stellar discs, tidal perturbations in the galactic gravitational field, gas clouds etc. Contrary to the hierarchical destruction, this process goes on up to now, and its efficiency is critically defined by the internal structure of the clumps. We will consider these processes in the present work and try to show that the clumps may be much less persistent than it is generally believed.

\section{ENERGY STRUCTURE OF CLUMPS}

It might appear at first sight that the difference between a cuspy and a cored profile is not that large, especially if the core is small. However, this conclusion is incorrect, if we take into account a possible velocity distribution anisotropy of DMPs. In order to illustrate it, we consider the evolution of DMP energy distribution during the halo formation. The halo is formed from a linear initial perturbation. The potential well of it was shallow. As a result, the initial total energies $\epsilon=\frac{v^{2}}{2}+\phi$ of the particles forming the halo were close: under very general assumptions, they lay in the interval $\epsilon \in\left[-1.5 \Phi_{\text {vir }} ;-\Phi_{\text {vir }}\right]$ (where $\Phi_{v i r}=G \frac{M_{v i r}}{R_{v i r}}$ ), i.e., they differed no more than 1.5 times [8]. The final potential well is much deeper. The ratio of its depth (i.e., the central potential $\phi(0))$ to the potential on the virial radius $-\Phi_{v i r}$ is $\phi(0) / \Phi_{v i r} \sim 30$ for the Milky Way [9] and even larger for smaller objects, since they should be more concentrated.

It is convenient to characterize each particle, instead of its energy, by its apocenter distance $r_{0}$, which is the largest distance that the particle can move away from the center. $r_{0}$ is defined by the particle energy and the shape of the potential well. For instance, if the particles had kept their initial energies from the interval $\left[-1.5 \Phi_{v i r} ;-\Phi_{v i r}\right]$, their $r_{0}$ would lie in $r_{0} \in\left[\frac{2}{3} R_{v i r} ; R_{v i r}\right]$.

A question appears: how strong can the energy evolution of the system during its formation be, i.e., can the relaxation be arbitrarily strong, or the ratio between the final $\epsilon_{f}$ and the initial $\epsilon_{i}$ energies of the particles is somehow limited for the majority of DMPs? In practice, two scenarios are possible:

1. Strong energy relaxation. The particle energies are completely redistributed, final particle energies cower all the potential well $\left[\phi(0) ;-\Phi_{v i r}\right]$. Then $r_{0}$ of the particles cover all the possible interval $\left[0, R_{v i r}\right]$ more or less evenly.

2. Moderate energy relaxation [8]. The final total specific energy $\epsilon_{f}$ of most of the particles differs from the initial ones $\epsilon_{i}$ no more than by a factor $k$

$$
\frac{\epsilon_{f}}{\epsilon_{i}} \leq k
$$

and $k \ll \phi(0) / \Phi_{\text {vir }}$. There can be particles that have changed their energy stronger, but their fraction is respectively small.

The relaxation is moderate if $k \leq c_{v i r} / 4$ [10]. This condition is very strict for galaxy clusters, but quite soft for low-mass galaxies with $c_{v i r}>20$ (though the real density profile may significantly differ from the NFW one, we used here the NFW halo concentration $c_{v i r}$ because of its popularity and in view of the fact that characteristic values of $c_{v i r}$ for various types of astronomical objects are well known).

The moderate relaxation leads to a very important consequence. Since initial particle energies were close to $-\Phi_{v i r}$ and changed no more than $k$ times during the halo formation, the halo contains only a small fraction of particles with the energies lower than $-k \Phi_{v i r}$ (it is important that $\left.\left|k \Phi_{\text {vir }}\right| \ll|\phi(0)|\right)$, which roughly corresponds to $r_{0} \simeq R_{v i r} / k$. Particles with more compact 
orbits are almost absent in the halo. It means that the density profile in the center of the halo, inside $R_{v i r} / k$, is mainly formed by the particles that arrive there from the outside. It leads to a very peculiar structure of the clump, differing drastically from the clumpy one.

First of all, it is easy to show that a cusp may form only in scenario 1, i.e., if the relaxation is strong $[8,10]$. If the relaxation is moderate, the density profile obligatory has a core. Nevertheless, the profile still can be very concentrated: the ratio of the core radius to the virial one can be arbitrarily small. The velocity distribution in the case of moderate relaxation is isotropic only in the very center of the halo [11] and gains some anisotropy with radius.

What scenario, 1 or 2 , is realized in nature? As of now, the answer to this question is not known with certainty. The mass hierarchy of the real Universe is so vast (from rich galaxy clusters of mass $\sim 10^{15} M_{\odot}$ down to clumps of mass $10^{-6} M_{\odot}$ or even lower) that it can be covered in no simulation, and a far interpolation is necessary. It makes discussible some simulation results concerning the clumps. For instance, if we interpolate the power-law grows of halo concentration $c_{v i r}$ with its mass decreasing [12], we obtain extremely large concentrations for clumps. This result is discussable [4]. Recent N-body simulations [13] suggest that the power-law grows of $c_{v i r}$ breaks for small-mass halos. Moreover, the simulations may suffer from underestimated numerical effects in halo centers [14]. N-body simulations favor strong energy relaxation leading to the cuspy profile. Their results nearly approximate galaxy clusters. However, if we consider smaller halos, like spiral galaxies or dwarf spheroidals, the model of moderate relaxation describes observations much better $[8,10]$. In particular, observations suggest cored profiles [15-20]. Therefore, we may expect that formation of low-massive clumps also follows the moderate relaxation scenario.

The moderate energy evolution can be characterized by the above-mentioned relaxation parameter $k$. As we will see in the Comparison of the predictions of core and cusp models section, the larger $k$ is, the stronger the relaxation is, and the more stable the clumps are. The clumps are the most fragile if $k=1$, i.e., when the clumps are formed with a negligible relaxation, and therefore their particles have $r_{0} \sim r_{c l}$. It is reasonable to expect that the moderate relaxation is much stronger $\left(k \simeq c_{v i r} / 4\right)[10]$. However, in this paper we will mainly consider the case $k=1$ for simplicity, as it gives the most pessimistic estimation of clump survival. Thus, though the choice between scenarios 1 and 2 is now unclear, the model we consider in this paper may be used to estimate the minimal value of the boost factor.

Moreover, if the assumption of the moderate energy evolution is true, it suggests that the majority of the particles still concentrate near $\epsilon=-\Phi_{\text {vir }}$ : the energy exchange of the particles during the relaxation is more or less a stochastic process, while the total energy of the system should conserve. Thus the narrowness of the ini-

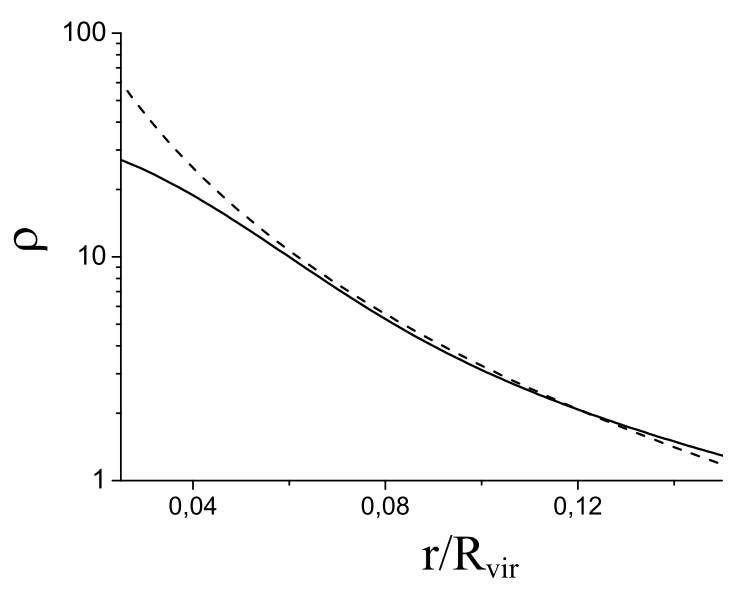

FIG. 1: The profile of a clump formed in the case if its energy relaxation was moderate (solid line). The NFW profile with the same concentration parameter $c_{v i r}=25$ is reproduced for comparison (dashed line). The density is represented in arbitrary units.

tial energy distribution, the relative smallness of the energy evolution, and the fact that the final potential well is much deeper, than the initial one, together result in crowding of the particle apocentre distances $r_{0}$ near $R_{v i r}$, i.e., $\left\langle r_{0}\right\rangle \sim r_{c l}$.

\section{TYPICAL CLUMP PARAMETERS}

If the dark matter particle is a WIMP of mass exceeding $100 \mathrm{GeV}$, the minimal clump mass is roughly equal to $10^{-6} M_{\odot}$, which corresponds to $R_{v i r} \sim 0.05 \mathrm{pc}$. The average density $\bar{\rho}$ of the clump is defined from $\bar{\rho} \cdot \frac{4}{3} \pi R_{v i r}^{3}=M_{v i r}$. We will use the following designations: $m_{6}=M_{v i r} / 10^{-6} M_{\odot}, r_{6}=R_{v i r} / 0.05 \mathrm{pc}$, $\bar{\rho}_{6}=1.9 \cdot 10^{-3} M_{\odot} / \mathrm{pc}^{3}$. For a clump with mass $M_{v i r}=10^{-6} M_{\odot}$ we have $m_{6}=r_{6}=\bar{\rho}_{6}=1$.

As we could see, almost all the particles in our model have $r_{0} \sim R_{v i r}$, and the dynamical time of the clump can be estimated as $t_{f} \sim \sqrt{\frac{R_{v i r}^{3}}{G M_{v i r}}} \propto(G \bar{\rho})^{-1 / 2}$. If $M_{v i r}=10^{-6} M_{\odot}, t_{f} \sim 10^{8}$ years. In the standard $\Lambda \mathrm{CDM}$ cosmology $\bar{\rho}$ depends on the clump mass only logarithmically [1, 10], and as a result, the clumps of all masses have comparable dynamical times. The time is significantly larger than typical times of any clump destruction mechanisms. Therefore, the clump disintegration can be considered in the instant tide approximation. 


\section{CLUMP DESTRUCTION: THE CASE OF THE MOST FRAGILE CLUMPS $k=1$}

There are several mechanisms of clump destruction in our Galaxy. We will consider only the most important ones. As we showed at the close of section Energy structure of clumps, the case $k=1$ corresponds to $r_{0} \sim r_{c l}$ and gives the most pessimistic estimation of clump survival. We will actively use this fact in the current section.

\section{A. Destruction on halo stars}

The clumps can be destroyed by collisions with stars. Let us denote the average star mass, star number density and star velocity dispersion by $m_{*}, n_{*}$, and $\sigma_{*}$, respectively. The dispersion of clump velocities is $\sigma_{c l}$. We may introduce $\sigma=\sqrt{\sigma_{*}^{2}+\sigma_{c l}^{2}}$. The characteristic time of clump destruction by collisions with stars is [21, eqn. $8.55]$

$$
t_{d} \simeq \frac{17}{200} \frac{\sigma m_{c l} r_{c l}^{2}}{G m_{*}^{2} n_{*} r_{0}^{3}},
$$

Since $r_{0} \sim r_{c l}$, we obtain:

$$
t_{d} \simeq \frac{17}{200} \frac{\sigma m_{c l}}{G m_{*}^{2} n_{*} r_{c l}},
$$

The main fraction of time the clumps spend in the Milky Way halo. The star concentration there can be estimated [7] as $n_{*}=\left(\rho_{h} / m_{*}\right)\left(r_{\odot} / r\right)^{3}$, where $r_{\odot}=8.5 \mathrm{kpc}, \rho_{h}=$ $1.4 \cdot 10^{-5} M_{\odot} / \mathrm{pc}^{3}, m_{*} \simeq 0.4 M_{\odot}$. We should integrate $n_{*}$ over the clump trajectory, from the minimum $r_{\text {min }}$ to the maximum $r_{\max }$ radius of its orbit. However, $n_{*}$ rapidly drops with radius, and the region near $r_{\text {min }}$ makes the main contribution into the clump destruction. Assuming $\sigma=200 \mathrm{~km} / \mathrm{s}$, we obtain from (4):

$$
t_{d} \simeq\left(r / r_{\odot}\right)^{3} m_{6}^{2 / 3} \cdot 8 \cdot 10^{10} \text { years. }
$$

As we can see, $t_{d}$ exceeds the age of the Universe even for low-massive objects. Only very small clumps $M_{v i r}<$ $10^{-7} M_{\odot}$ can be destroyed by the halo star collisions.

\section{B. Destruction on the Milky Way disc}

However, the clumps can be disrupted by the tidal perturbations of the galactic disc as a whole. A single passing of a clump through the disc changes its energy on

$$
\delta E=\frac{16 \pi^{2} G^{2} o_{d}^{2} r_{0}^{2}}{3 v_{\perp}^{2}},
$$

where $o_{d}$ is the surface density of the Milky Way disc, $v_{\perp}$ is the perpendicular to the disc component of the clump velocity. We assume [7]

$$
o_{d}(r)=\frac{M_{d}}{2 \pi r_{d}^{2}} e^{-r / r_{d}},
$$

with $M_{d}=8 \cdot 10^{10} M_{\odot}, r_{d}=4.5 \mathrm{kpc}$. Dividing the total clump energy $|E| \sim \frac{G m_{c l}}{r_{c l}}$ by (6), we obtain the number of passing $N_{d}$ needed to destroy the clump by the disc

$$
N_{d}=\frac{3 r_{d}^{4} v_{\perp}^{2}}{8 G M_{d}^{2}} \frac{m_{c l}}{r_{c l}^{3}} e^{2 r / r_{d}} .
$$

Substituting[33] here $v_{\perp} \sim 100 \mathrm{~km} / \mathrm{s}$, we obtain $N_{d} \simeq$ $4.5 \cdot 10^{-4} \bar{\rho}_{6} e^{2 r / r_{d}}$. As we can see, $N_{d}$ depends only on the average density of the clump. Since $\bar{\rho}$ depends on the clump mass only logarithmically in the standard $\Lambda \mathrm{CDM}$ model $[1,10]$, the clump destruction by the disc depends almost not at all on the clump mass.

The motion of a clump in the Galaxy can be characterized by the minimum $r_{\min }$ and the maximum $r_{\max }$ radii of its orbit. Equation (8) shows that a single passing through the disk is enough to disintegrate a clump if $r_{\min }<17 \mathrm{kpc}$. So high efficiency of the mechanism under consideration in the central area of the Milky Way justifies our neglect of other clump destruction factors, like the galactic bulge, gas nebulas etc. In the regions where they are effective, the clumps are disrupted anyway.

On the other hand, the disc surface density rapidly drops with radius, and so does the efficiency of clump destruction. A clump passes through the disc twice in each revolution. Let us consider a clump with a circular orbit of radius $25 \mathrm{kpc}$. The orbital period of the Solar System about Galactic Center is $T_{M W} \sim 230 \mathrm{Myr}$. If we accept that the Milky Way density profile is $\rho \propto r^{-2}$ (which is a very rough approximation), $T \propto r$ [22], i.e., the orbital period of the clump is $T \simeq 230 \mathrm{Myr} \cdot 25 \mathrm{kpc} / 8 \mathrm{kpc} \simeq$ 700 Myr. The clump passed through the disc $\sim 30$ times in the lifetime of the Galaxy. According to Eqn. 8, the clump should be destroyed in $\sim 30$ passages. Thus $r=25 \mathrm{kpc}$ is approximately the border of the region where the clumps were disrupted by the disc in the lifetime of the Galaxy. Clumps with larger $r$ also can be destroyed, if they have an elongate orbit and pass through the disc close to the center. However, the fraction of these clumps falls approximately as $r^{-2}$ and becomes negligible at $r \sim 50 \mathrm{kpc}$.

\section{Destruction by the gravitational field of the Galaxy}

If a clump has a non-circular orbit, it periodically approaches the galaxy center and expresses tidal shocks. A single shock increases the clump energy on [21, eqn. 8.43]

$$
\delta E=\frac{4 G^{2} M_{p}^{2}\left(r_{\min }\right)}{3 v^{2} r_{\min }^{4}}\left\langle r_{0}^{2}\right\rangle,
$$

where $v$ is the clump velocity at $r_{\text {min }}$, and $M_{p}\left(r_{\text {min }}\right)$ is the total mass of the perturber (in our case, of the Milky Way) inside $r_{m i n}$, and $\left\langle r^{2}\right\rangle$ is the mass-weighted mean-square radius of the clump. If the clump density 
profile is $\rho \propto r^{-2}$ and $r_{0}=r_{c l},\left\langle r^{2}\right\rangle=r_{0}^{2} / 3=r_{c l}^{2} / 3$. Though a real clumps apparently have other profiles, we may use approximation $\left\langle r^{2}\right\rangle=r_{c l}^{2} / 3$ in our estimative calculations. Dividing the clump energy $E \simeq G m_{c l} / r_{c l}$ by $\delta E$, we obtain the number of rotations necessary to destroy the clump

$$
N_{d}=\frac{9}{4}\left(\frac{v^{2} r_{\min }}{G M_{p}\left(r_{\min }\right)}\right)\left(\frac{m_{c l}}{M_{p}\left(r_{\min }\right)}\right)\left(\frac{r_{\min }}{r_{c l}}\right)^{3} .
$$

If the orbit has a significant eccentricity, we may estimate $v^{2} \simeq G M_{p}\left(r_{\text {min }}\right) / r_{\text {min }}$.

$$
N_{d}(r) \simeq 2 \frac{\bar{\rho}_{c l}}{\bar{\rho}_{p}(r)},
$$

where $\bar{\rho}_{p}(r)$ is the average density if the perturber inside radius $r$. Thus the clump can be effectively destroyed if it passes through the areas where its average density is comparable with the density of the perturber. The destruction process again depends only on $\bar{\rho}_{c l}$ and therefore is almost insensitive to the clump mass. According to equation (11), a clump should have orbit radius $r \sim 18 \mathrm{kpc}$ do be destroyed in one turn about the Galaxy center and $r \sim 50 \mathrm{kpc}$ to be destroyed in the lifetime of the Galaxy, for the conventional Milky Way models.

We should underline that the mechanism efficiency strongly depends on the orbit eccentricity: a clump on a circular or near-circular orbit expresses no shocks. However, there is a clump destruction mechanism that is not sensitive at all to the shape of the clump orbit. Indeed, let us consider a particles belonging to clump that rotates around its center having the apocenter distance $r_{0}$. The tidal force from the Galaxy gravitational field can be estimated as $\left(G M_{p}(r) / r^{2}\right) \cdot\left(r_{0} / r\right)$ in the framework of the clump center, where $r$ is the clump distance from the Galaxy center. The work of the force on the particle orbit is $\sim r_{0} \cdot\left(G M_{p}(r) / r^{2}\right) \cdot\left(r_{0} / r\right)$. If it is comparable with the bind energy of the particle $\sim G m_{c l} / r_{0}$, the particle can be teared out of the clump. We assume $r_{0} \sim r_{c l}$, as we did before. The condition of clump destruction is

$$
\frac{M_{p}(r)}{r^{3}}>\frac{m_{c l}}{r_{c l}^{3}} \quad \text { or } \quad \bar{\rho}_{c l}<\bar{\rho}_{p}(r) .
$$

If we compare equation (11) with the last one, we can see, that it is approximately equal to the condition for a clump to be destroyed in one or two turns around the Galaxy center. Thus the direct tidal disruption is in general less effective than the tidal shocks. On the other hand, equation (12) guarantees that all the clumps (in the most pessimistic case for their survival, when $r_{0} \sim r_{c l}$ ) are destroyed inside $r \sim 20 \mathrm{kpc}$, disregarding their orbits.

To summarize: The clumps in the Milky Way halo are destroyed by the disc and by tidal effects in the Galaxy gravitational field. The efficiency of these two mechanisms is comparable. Collisions with halo stars are not effective. All the clumps should be destroyed inside $\sim 20 \mathrm{kpc}$ from the Galaxy center (we should remind that we consider the most pessimistic scenario). A gray zone lies between 20 and $50 \mathrm{kpc}$, some clumps should be destroyed there, some may survive. No mechanism effectively disrupts clumps out of $50 \mathrm{kpc}$ from the Milky Way center, even under the most pessimistic assumptions about the inner clump structure that we consider.

\section{Dwarf spheroidals. Segue 1.}

Among all astrophysical objects containing dark matter, dwarf satellites of the Local Group draw special attention. They are very promising for detecting darkmatter annihilation. Though the dark-matter density in these objects is significantly lower than that at the Galactic center, they typically contain no sources of cosmic rays, and therefore almost any high-energy signal detected from the dwarf galaxies could be the signal of dark-matter annihilation.

Segue 1 draws the most attention among numerous satellites in the Local Group [23]. It combines several advantages: it is the closest satellite to Earth, it is located at high Galactic latitude (and, consequently, superposes on a low gamma-ray background) and expected to produce the strongest dark-matter signal [24], though the last statement is still discussable. Recent observations can essentially constrain the most probable WIMP dark matter models (with the cross-section corresponding to the dark-matter abundance $\langle\sigma v\rangle_{a} \simeq 3 \cdot 10^{-26} \mathrm{~cm}^{3} / \mathrm{s}$ and DMP mass range that has not been excluded by LHC experiments) only if the dwarf spheroidals are expected to have a significant boost factor [25]. Therefore the question of the substructures in dwarf spheroidals is extremely important.

Segue 1 has the highest known mass-to-light ratio of any observed galaxy, which implies that Segue 1 is strongly dominated by dark matter. Its density distribution can be modelled by the Einasto profile

$$
\rho=\rho_{e} \exp \left[-2 n\left\{\left(\frac{r}{r_{e}}\right)^{\frac{1}{n}}-1\right\}\right]
$$

with $\rho_{e}=0.11 M_{\odot} \mathrm{pc}^{-3}, r_{e}=150 \mathrm{pc}$, and $n=3.3$ [26]. Unfortunately, all the parameters are poorly known and contain huge uncertainties: all the stars $(\simeq 66)$ observed in Segue 1 lie inside $10^{\prime}(\simeq 67 \mathrm{pc}$ ) from the center, which is two times smaller than $r_{e}$. For instance, a completely different set of parameters $\rho_{e}=0.094 M_{\odot} \mathrm{pc}^{-3}, r_{e}=$ $70 \mathrm{pc}$ fits the observations equally well [27].

Apparently, Segue 1 does not contain a disc. However, the clumps could still be disrupted by stars or by the tides in the gravitational field of the dwarf. We consider both these mechanisms.

Segue 1 contains approximately a thousand stars [28] that are situated inside $\sim 67 \mathrm{pc}$ from the center. We may very roughly estimate $n_{*} \simeq 8 \cdot 10^{-4} \mathrm{pc}^{-3}$. Substituting $m_{*}=0.4 M_{\odot}$ and $\sigma=60 \mathrm{~km} / \mathrm{s}$ (which is approximately the escape velocity from the center of Segue $1[25]$ ), we 
obtain

$$
t_{d} \simeq m_{6}^{2 / 3} \cdot 10^{9} \text { years. }
$$

As we can see, the smallest clumps are destroyed by the stars. However, clumps of mass $m_{6}>30$, i.e., $m>$ $3 \cdot 10^{-5} M \odot$, cannot be disrupted by this mechanism.

In contrast, the clump destruction by the gravitational field of Segue 1 is extremely effective. We can see from eqn. (13) that the equality $\bar{\rho}_{c l}=\bar{\rho}_{p}(r)$ occurs at the distance $\sim 1300$ pc from the center of Segue 1 (if we suppose that mass distribution (13) is still valid there).

\section{THE CASE OF ROBUST CLUMPS $k=c_{v i r} / 4$}

The case of $k=1$ is extreme and not very probable, even if the relaxation is moderate. One may expect that $k$ depends on $c_{v i r}$ of the halo. The most probable value of $k$ is $c_{v i r} / 4$ [10], which is close to the maximum value of $k$, at which the relaxation may still be named moderate. Therefore we should consider the case $k=c_{v i r} / 4$.

First of all, now $k$ depends on $c_{v i r}$. If $k=c_{v i r} / 4$, $\left\langle r_{0}\right\rangle \gtrsim r_{c l} / k=4 R_{v i r} / c_{v i r}$ [8], and we should substitute this value to (9) and (6). Recent simulation suggest that the power-law growth of $c_{v i r}$ with the halo mass decreasing breaks, and the concentration of low-mass objects grows much slower. A sophisticated analysis [13] has shown that even the smallest halos have $c_{\text {vir }} \simeq 60$. We will use this value as the most plausible.

Equation (8) for the number of rotations necessary to destroy the clump by the Milky Way disc transforms into

$$
N_{d}=\frac{3 r_{d}^{4} v_{\perp}^{2} c_{v i r}^{2}}{128 G M_{d}^{2}} \frac{m_{c l}}{r_{c l}^{3}} e^{2 r / r_{d}} .
$$

A reasoning similar to that used below equation (8) shows that $r=15 \mathrm{kpc}$ is approximately the border of the region where the clumps were disrupted by the disc in the lifetime of the Galaxy in the case of robust cored clumps with $k=c_{v i r} / 4$. This value is substantially smaller than that for the most fragile clumps $k=1$, but larger then the value obtained for the cuspy clumps $r \sim 8 \mathrm{kpc}$ [7]. Thus the durability of the cored clumps may vary in a wide range. However, an essential difference between cuspy and cored models remains.

Equation (12) transforms into

$$
\frac{c_{v i r}^{2}}{16} \rho_{c l}<\bar{\rho}_{p}(r) .
$$

This equation shows that the dwarf satellites like Segue 1 are so dense that it is difficult to expect that any cored clumps could survive in it. Indeed, if we accept $c_{v i r} \simeq 60$ for clumps, then the clumps are destroyed in the region where $\bar{\rho}_{p}(r)>225 \rho_{c l}$. Equation (13) shows that all the clumps should be destroyed inside $\sim 2 r_{e}$, i.e., inside $300 \mathrm{pc}$ from the center of Segue 1.

The conclusion of absence of any DM substructures is probably valid for all dwarf satellites of the Local Group.

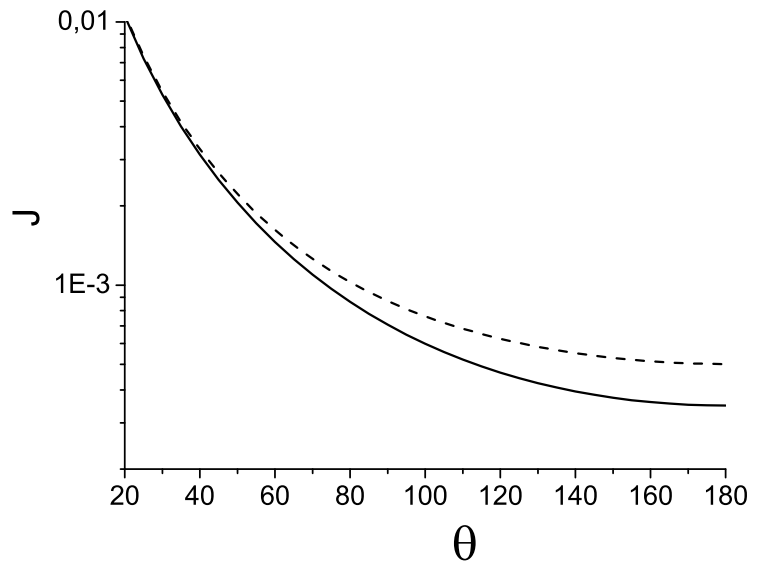

FIG. 2: The annihilation signal $J$ from the Milky Way as a function of the angle $\theta$ between the observation line and the direction towards the Galaxy center. The normalization of $J$ is arbitrary. The solid line corresponds to the case when the clumpy structure disappears at $r_{\text {destr }}=50 \mathrm{kpc}$, the dashed line - to $r_{\text {destr }}=10 \mathrm{kpc}$. The boost factor at $50 \mathrm{kpc}$ is set to be equal to $C_{50}=20$.

The satellites, as we observe them now, are the most probably the central and the densest parts of ancient dwarf galaxies tidally destroyed by the Milky Way and M31. As a result, their density is very high, and the clumps are easily destroyed when approaching their centers. We should also take into account that the tidal perturbations destroying the satellites disrupted their clump structure as well.

\section{COMPARISON OF THE PREDICTIONS OF CORE AND CUSP MODELS}

Now we can compare the survival of the cored clumps formed under the assumption of moderate relaxation (hereafter MR-clumps) with one of the standard model NFW clumps $[5,7]$. As we can see, clumps are destroyed much easier in our case.

This result should not surprise. The clumps of the standard model are significantly more stable not because of the existence of cusp per se: MR-clumps can also be very concentrated and have large central density (Fig (1)). The principle difference is the particle orbits. The particles forming the cusp in the NFW model are confined in the cusp: they have small quasi-circular orbits with the apocenter distance $r_{0} \simeq 0$. According to eqn. 6 , the energy perturbations are proportional to $r_{0}^{2}$. This fact along provides the cusp stability. Moreover, the particles with $r_{0} \simeq 0$ have a very short dynamical time, the tidal perturbations are adiabatic for these particles, and equations like (8) or (11) are not valid anymore. The adiabatic invariant conservation makes the cusp extremely resistant to tidal perturbations. A NFW clump 
easily looses its outer layers, but the cusps are almost immune. They can be destroyed only in the bulge of the Milky Way, at the distances less then $\sim 3 \mathrm{kpc}$ from the Galaxy center [7], and most likely survive in the dwarf spheroidals. Since it is cusps that makes the main contribution into the boost factor, it should be significant in the standard model.

The MR-clumps are built completely differently. They still can have a very high central density, and their profile can be relatively close to the NFW, except of a small central region, where they have cores (see Fig (1)). However, the majority of the DMPs have the apocenter radius $r_{0}$ comparable with the virial radius of the clump. It is true even for the core itself: The bulk of the particles only 'visit' the halo center, having $r_{0} \gg r_{c}$, where $r_{c}$ is the core radius. As we could see, the particles with large apocenter distance $r_{0}$ are much easier for the tidal effects to detach. The MR-clumps have a much larger apocenter radius $\left\langle r_{0}\right\rangle$ and therefore are much easier to be destroyed.

Evidently the largest possible value $\left\langle r_{0}\right\rangle=R_{v i r}$, which corresponds to the relaxation parameter $k=1$. It is exactly the assumption we used in obtaining of the results of this paper. Therefore we have obtained the low limit on the clump stability. The most probable value of $k$ is $k \simeq c_{v i r} / 4$, where $c_{v i r}$ is the NFW halo concentration [10]. Then $\left\langle r_{0}\right\rangle \simeq 2 R_{v i r} / c_{v i r}$, and the clump survival is somewhat higher. However, it is still much lower for the cored clumps than for the cuspy ones.

We have shown that the clump survival strongly depends on their internal structure, which is not quite clear now. Since the annihilation signal rapidly increases with the DM density growth, compact and dense DM regions attract the main attention of the indirect DM searches. Our calculations show that the clump structure in these objects can be utterly destroyed under quite natural cosmological assumptions.

It is important that, even under the most pessimistic suppositions about their structure, the clumps in the Milky Way should survive at the distances exceeding $50 \mathrm{kpc}$ from the Galaxy center. It means that the clumpy structure should survive in almost all the dark matter in the Universe. If we consider the Local Group, 80\% of its DM containment form the halos of the Milky Way and M31 outside $50 \mathrm{kpc}$ from their centers and probably a common DM envelope of the system [29]. Broadly speaking, the main part of the dark matter in the Universe forms low-density structures, like DM envelopes of galaxy clusters, walls and filaments of the large scale structures etc. All this dark matter should have kept entirely its clumpy structure. This fact is very important for the estimations of the extragalactic background, possibly created by the DM annihilation.

\section{PHENOMENOLOGY: PREDICTIONS OF THE ANNIHILATION SIGNAL AND UNCERTAINTIES}

We should underline that the phenomenological consequence of the DM substructures is not only the annihilation signal boosting by some factor. The signal dependence on the DM density $\rho$ changes. If the dark matter is homogeneous, the annihilation signal is proportional to $\rho^{2}$. If the annihilation in clumps dominates, the signal is proportional to the number density of the clumps, i.e., to $\rho$. Thus, if the clumps exist in the present-day DM structures, the DM annihilation should be boosted, and the boost factor should be roughly proportional to $\rho^{-1}$.

Let us discuss the uncertainties and possible observable consequences of the model. As we have shown, the clumps survive outside of $50 \mathrm{kpc}$ from the galactic center even under the most pessimistic supposition about their structure. On the other hand, [7] showed that even rather robust NFW clumps are strongly destroyed closer than $8 \mathrm{kpc}$ from the center. We may conclude that $20-50 \mathrm{kpc}$ is quite a reliable estimation of the maximum distance from the Milky Way center where the cored clumps can still be destroyed. The boost factor estimations are much more ambiguous.

\section{A. A sketch of the standard theory of the boost factor}

If the comoving number density of halos in the mass range of $[M ; M+d M]$ at the redshift $z$ is $d p(M, z) / d M$, the boost factor of the system $C$ can be represented as a multiplication (see details in [30]):

$$
C=\Delta \cdot F_{\text {coll }}(z) \cdot\left[B_{\text {halo }}\right]
$$

Here $\Delta$ is the mean halo overdensity defined by the cosmological model, of a halo in units of the cosmic mean matter density, $F_{\text {coll }}(z)=\int \frac{d p}{d M} M d M /\langle\rho\rangle$ is the mass fraction collapsed into cosmological halos at $z(\langle\rho\rangle$ is the average density of the Universe). $B_{\text {halo }}$ is a single halo clumping factor $B_{\text {halo }}=\int\left(\frac{\rho}{\langle\rho\rangle_{\text {halo }}}\right)^{2} d^{3} r / \int d^{3} r$, where $\langle\rho\rangle_{\text {halo }}$ is the halo average density, and $\left[B_{\text {halo }}\right] \equiv$ $\int B_{\text {halo }} \frac{d p}{d M} M d M / \int \frac{d p}{d M} M d M$.

As we can see, the boosting is determined by three functions: the halo mass function $p(M, z)$, the boost for a single halo $B_{\text {halo }}$ as a function of the halo concentration $c_{v i r}$, and the concentration dependence $c_{v i r}(M, z)$ of halo mass and $z$.

$$
B_{\text {halo }}=\frac{c_{v i r}^{3}\left(1-1 /\left(1+c_{v i r}\right)^{3}\right)}{9\left(\ln \left(1+c_{v i r}\right)-c_{v i r} /\left(1+c_{v i r}\right)\right)^{2}}
$$

for the case of NFW halos. 


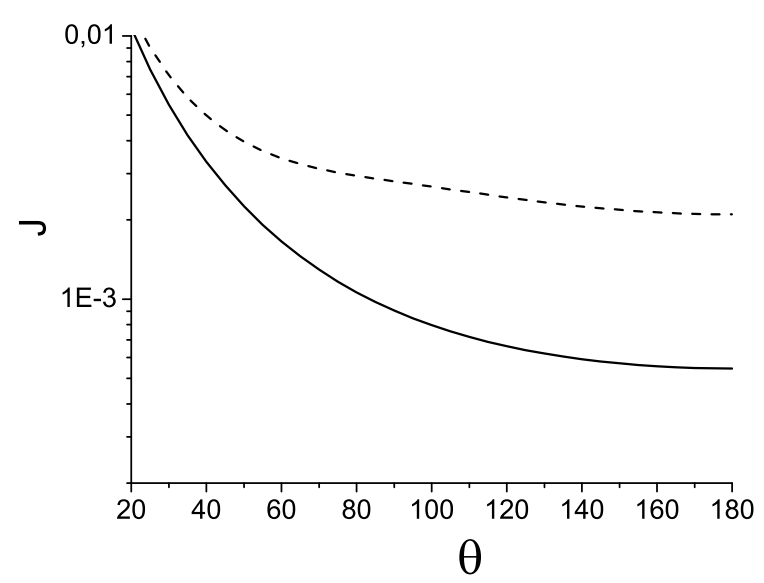

FIG. 3: The annihilation signal $J$ from the Milky Way as a function of the angle $\theta$ between the observation line and the direction towards the Galaxy center. The normalization of $J$ is arbitrary. The solid line corresponds to the case when the clumpy structure disappears at $r_{\text {destr }}=50 \mathrm{kpc}$, the dashed line - to $r_{\text {destr }}=10 \mathrm{kpc}$. The boost factor at $50 \mathrm{kpc}$ is set to be equal to $C_{50}=100$.

\section{B. The boosting in the case of cored clumps}

What changes in the picture, if we consider the abovestated model instead of the traditional NFW one? It is surprising that the mass function should be almost the same for the cuspy and cored clumps. Indeed, two main factors defining $p(M, z)$ are the spectrum of the initial cosmological perturbations and the clump destruction during the hierarchical structure formation. The first factor has nothing to do with the profiles of formed clumps. The second process (in the framework of the standard $\Lambda \mathrm{CDM}$ cosmology) is also not sensitive to the clump density profile. The objects of different masses collapse almost simultaneously in $\Lambda \mathrm{CDM}$, and, as we have already mentioned, the hierarchical destruction time is shorter than the virilization time. Therefore, the approach used by [5] and considering non-virialized clumps is exactly valid for in our case and leads to the same mass function.

The only mechanism that significantly changes $d p(M, z) / d M$ and essentially depends on the clump density profile is the astrophysical clump destruction. Let us consider the Local Group. As we could see, the clumps situated farther than $50 \mathrm{kpc}$ from the Milky Way center should survive under the most pessimistic assumptions about their structure. However, $\sim 70 \%$ of the Milky Way dark matter lies outside of this radius [31], and we may suppose that the same situation takes place in the Andromeda galaxy. Moreover, along with the DM haloes of large and dwarf galaxies, the Local Group may contain an approximately equal quantity of dark matter that is not bound in the galaxies and presumably forms a large envelope $[11,21,29]$. Thus, $\sim 85 \%$ of the Local Group ut-

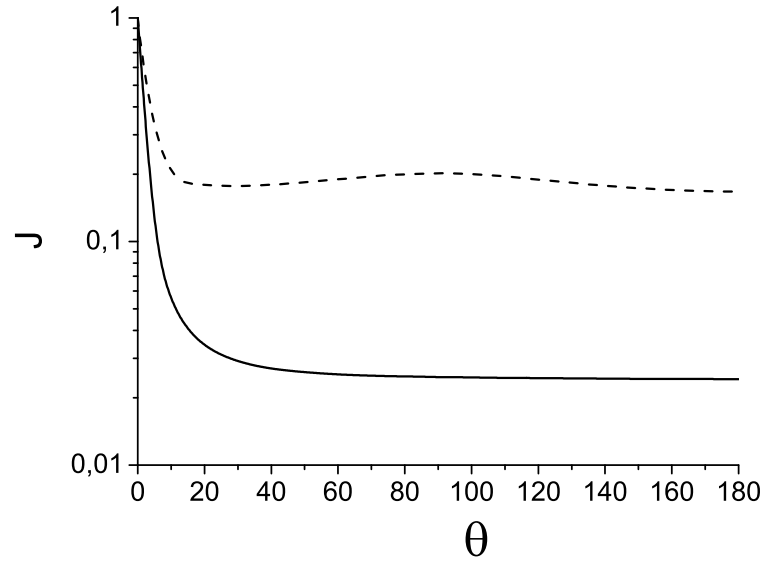

FIG. 4: The annihilation signal $J$ from the Milky Way as a function of the angle $\theta$ between the observation line and the direction towards the Galaxy center. The normalization of $J$ is arbitrary. The solid line corresponds to the case when the clumpy structure disappears at $r_{\text {destr }}=50 \mathrm{kpc}$, the dashed line - to $r_{\text {destr }}=10 \mathrm{kpc}$. The boost factor at $50 \mathrm{kpc}$ is set to be equal to $C_{50}=10^{4}$.

terly kept its clumpy structure, and if we consider a large area of dark matter, significantly exceeding the maximum halo size, the modification of the averaged $d p(M, z) / d M$ is not very significant.

Of course, $B_{\text {halo }}$ depends on the clump density profile. However, even in the case of the NFW clump with infinite central density, the annihilation signal $\left(\propto \rho^{2} \cdot 4 \pi r^{2} \propto\right.$ const) has no peculiarity in the center. Since the clear discrepancy between cores and cusps occurs only at $r \ll$ $r_{s}, B_{\text {halo }}$ for these cases may differ only on a factor $\lesssim$ 2 (see minimal model in [25] as an illustration). Thus the difference in value of $B_{\text {halo }}$ between cored and cuspy clumps is much less then the uncertainties of $c_{v i r}(M, z)$.

Indeed, the uncertainty of $c_{v i r}(M, z)$ (especially the dependence on $M$ ) is the weakest point of any DM indirect search theory. Since $d p(M, z) / d M$ rapidly drops with $M$, the preponderant contribution into $\left[B_{\text {halo }}\right]$ is given by the smallest clumps, and we need to know their concentration. Typically $c_{v i r}(M, z)$ is defined from the results of N-body simulations that suggest a power-law dependence [30]. However, the ratio of masses of the smallest halos with properly resolved density profiles to the largest ones hardly exceeds $3-4$ orders of magnitude even in recent large simulations containing $\sim 10^{9}$ test bodies. The mass hierarchy of the real Universe covers at least 21 orders of magnitude. Therefore, the estimations of $c_{v i r}$ for the smallest clumps are based on a far interpolation. It is questionable whether the power-law behavior of $c_{v i r}(M, z)$ holds within such a vast range of masses [4]. Recent N-body simulations suggest that $c_{v i r}<70$ even for the smallest clumps [13]. Moreover, $B_{\text {halo }} \propto c_{v i r}^{3}$ if $c_{v i r} \gg 1$, i.e., $B_{\text {halo }}$ is sensitive even to small changes of $c_{v i r}$. All these facts make the predictions of $\left[B_{h a l o}\right]$ very 
dependent on the suppositions of the $c_{v i r}(M, z)$ behavior. Now we will discuss several possible scenarios by the example of the Milky Way.

Thus, if we consider a large area of dark matter, significantly exceeding the maximum halo size, the difference between cuspy and cored scenarios can be surprisingly small, especially if we take into account the large unavoidable uncertainties. For instance, if we could measure the extragalactic background produced by the DM annihilation, we would be unable to choose between the scenarios because of our ignorance about $c_{v i r}(M, z)$ of the small clumps. However, if we consider a separate halo, the predictions of the models can be drastically different.

In order to illustrate this, we consider the Milky Way halo, as it is a popular object for the indirect DM search. For simplicity, we assume that the clumpy structure appears stepwise at some radius $r_{\text {destr }}$ : there is no substructure inside this radius, and the substructures utterly survive outside of $r_{\text {destr }}$. In order to take into account the uncertainties of $c_{v i r}$ determination, we presume the total boost factor $C_{50}$ at $r=50 \mathrm{kpc}$ from the Galaxy center. The absolute value of the signal depends on the particle nature of the $\mathrm{DM}$, and we choose the normalization arbitrarily (but the same for all the cases). The figures (2-4) show the annihilation signal $J$ from the Milky Way as a function of the angle $\theta$ between the observation line and the direction towards the Galaxy center. The solid line corresponds to $r_{\text {destr }}=50 \mathrm{kpc}$, which is roughly consistent with the scenario of the least stable cored clumps, the dashed line - to $r_{\text {destr }}=10 \mathrm{kpc}$, which approximately models the NFW clumps [7]. Fig. 2, 3, 4 correspond to $C_{50}=20,100,10^{4}$, respectively.

As we can see, the difference between the scenarios is not significant, if the boosting is not very strong $\left(C_{50}=\right.$ 20, Fig. 2). The dashed line on Fig. 3 approximately coincides with Fig. 5 in [7]: the model with $r_{\text {destr }}=10 \mathrm{kpc}$ and $C_{50}=100$ indeed corresponds to that considered in this paper and assuming the NFW clumps and holding the power-law behavior of $c_{v i r}(M)$ obtained in N-body simulations. The cases of $C_{50}=20$ and $C_{50}=10^{4} \mathrm{de}-$ scribe slower and faster grows of $c_{v i r}(M)$, respectively. One can see that the difference between the cuspy and cored clumps becomes quite significant if $C_{50} \geq 100$ : the external regions of the halo generate a bright background, and it is significantly dimer if the clumps are easy to be destroyed. The background significantly modifies the angular profile of the signal. However, even if the boost factor is as large as $C_{50}=10^{4}$, the background produced by the outer clumpy regions of the halo is not bright enough to overshadow the Galaxy center.

\section{Acknowledgments}

Financial support by Bundesministerium für Bildung und Forschung through DESY-PT, grant 05A11IPA, is gratefully acknowledged. BMBF assumes no responsibility for the contents of this publication. We acknowledge support by the Helmholtz Alliance for Astroparticle Physics HAP funded by the Initiative and Networking Fund of the Helmholtz Association. The work is supported by the CONICYT Anillo project ACT-1122 and the Center of Excellence in Astrophysics and Associated Technologies (PFB06).
[1] D. S. Gorbunov and V. A. Rubakov, Introduction to the Early Universe theory. Volume 2: Cosmological perturbations. LKI publishing house, Moscow, 2010.

[2] K. P. Zybin, M. I. Vysotsky, and A. V. Gurevich, The fluctuation spectrum cut-off in a neutralino dark matter scenario., Physics Letters A 260 (Sept., 1999) 262-268.

[3] S. Hofmann, D. J. Schwarz, and H. Stöcker, Damping scales of neutralino cold dark matter, Phys. Rev. D 64 (Oct., 2001) 083507, [\{astro-ph/0104173\}].

[4] D. Anderhalden and J. Diemand, Density profiles of CDM microhalos and their implications for annihilation boost factors, JCAP 4 (Apr., 2013) 9, [\{arXiv: 1302.0003\}].

[5] V. Berezinsky, V. Dokuchaev, and Y. Eroshenko, Destruction of small-scale dark matter clumps in the hierarchical structures and galaxies, Phys. Rev. D $\mathbf{7 3}$ (Mar., 2006) 063504, [\{astro-ph/0511494\}].

[6] J. Diemand, B. Moore, and J. Stadel, Earth-mass dark-matter haloes as the first structures in the early Universe, Nature (London) 433 (Jan., 2005) 389-391, [ astro-ph/0501589\}].

[7] V. Berezinsky, V. Dokuchaev, and Y. Eroshenko, Remnants of dark matter clumps, Phys. Rev. D $\mathbf{7 7}$
(Apr., 2008) 083519, [\{arXiv:0712.3499\}].

[8] A. N. Baushev, Galaxy Halo Formation in the Absence of Violent Relaxation and a Universal Density Profile of the Halo Center, Astrophys. J. 786 (May, 2014) 65, [\{arXiv: 1205.4302\}].

[9] L. S. Marochnik and A. A. Suchkov, The Galaxy. 1984.

[10] A. N. Baushev, Relaxation of dark matter halos: how to match observational data?, A 6 A 569 (Sept., 2014) A114, [\{arXiv:1309.5162\}].

[11] A. N. Baushev, Extragalactic Dark Matter and Direct Detection Experiments, Astrophys. J. 771 (July, 2013) 117, [\{arXiv:1208.0392\}].

[12] J. F. Navarro, A. Ludlow, V. Springel, J. Wang, M. Vogelsberger, S. D. M. White, A. Jenkins, C. S. Frenk, and A. Helmi, The diversity and similarity of simulated cold dark matter haloes, MNRAS 402 (Feb., 2010) 21-34, [\{arXiv:0810.1522\}].

[13] M. A. Snchez-Conde and F. Prada, The flattening of the concentrationmass relation towards low halo masses and its implications for the annihilation signal boost, Monthly Notices of the Royal Astronomical Society 442 (2014), no. 3 2271-2277, [ http://mnras.oxfordjournals.org/content/442/3/ 
2271.full.pdf+html\}].

[14] A. N. Baushev, The real and apparent convergence of $N$-body simulations of the dark matter structures: Is the Navarro-Frenk-White profile real?, Astroparticle Physics 62 (Mar., 2015) 47-53, [\{arXiv:1312.0314\}].

[15] W. J. G. de Blok, S. S. McGaugh, and V. C. Rubin, High-Resolution Rotation Curves of Low Surface Brightness Galaxies. II. Mass Models, AJ 122 (Nov., 2001) 2396-2427.

[16] W. J. G. de Blok and A. Bosma, High-resolution rotation curves of low surface brightness galaxies, $A \mathscr{E} A$ 385 (Apr., 2002) 816-846, [\{astro-ph/\}].

[17] D. Marchesini, E. D'Onghia, G. Chincarini, C. Firmani,

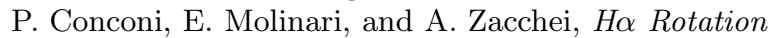
Curves: The Soft Core Question, Astrophys. J. $\mathbf{5 7 5}$ (Aug., 2002) 801-813, [\{astro-ph/\}].

[18] G. Gentile, P. Salucci, U. Klein, and G. L. Granato, $N G C$ 3741: the dark halo profile from the most extended rotation curve, MNRAS 375 (Feb., 2007) 199-212, [ astro-ph/\}].

[19] L. Chemin, W. J. G. de Blok, and G. A. Mamon, Improved Modeling of the Mass Distribution of Disk Galaxies by the Einasto Halo Model, AJ 142 (Oct., 2011) 109, [\{arXiv:1109.4247\}].

[20] S.-H. Oh, W. J. G. de Blok, E. Brinks, F. Walter, and R. C. Kennicutt, Jr., Dark and Luminous Matter in THINGS Dwarf Galaxies, AJ 141 (June, 2011) 193, [\{arXiv: 1011.0899\}].

[21] J. Binney and S. Tremaine, Galactic Dynamics: Second Edition. Princeton University Press, 2008.

[22] A. N. Baushev, Principal properties of the velocity distribution of dark matter particles on the outskirts of the Solar system, MNRAS 417 (Oct., 2011) L83-L87, [\{arXiv:1103.3828\}].

[23] A. Geringer-Sameth, S. M. Koushiappas, and M. G. Walker, Comprehensive search for dark matter annihilation in dwarf galaxies, Phys. Rev. D 91 (Apr., 2015) 083535, [\{arXiv:1410.2242\}].

[24] R. Essig, N. Sehgal, L. E. Strigari, M. Geha, and J. D. Simon, Indirect dark matter detection limits from the ultrafaint Milky Way satellite Segue 1, Phys. Rev. D 82 (Dec., 2010) 123503, [\{arXiv:1007.4199\}].

[25] A. N. Baushev, S. Federici, and M. Pohl, Spectral analysis of the gamma-ray background near the dwarf Milky Way satellite Segue 1: Improved limits on the cross section of neutralino dark matter annihilation, Phys. Rev. D 86 (Sept., 2012) 063521, [\{arXiv: 1205.3620\}].

[26] J. Aleksić, E. A. Alvarez, L. A. Antonelli, P. Antoranz, M. Asensio, M. Backes, J. A. Barrio, D. Bastieri, J. Becerra González, W. Bednarek, A. Berdyugin, K. Berger, E. Bernardini, A. Biland, O. Blanch, R. K. Bock, A. Boller, G. Bonnoli, D. Borla Tridon, I. Braun, T. Bretz, A. Cañellas, E. Carmona, A. Carosi, P. Colin, E. Colombo, J. L. Contreras, J. Cortina, L. Cossio, S. Covino, F. Dazzi, A. De Angelis, E. De Cea del Pozo, B. De Lotto, C. Delgado Mendez, A. Diago Ortega, M. Doert, A. Domínguez, D. Dominis Prester, D. Dorner, M. Doro, D. Elsaesser, D. Ferenc, M. V. Fonseca, L. Font, C. Fruck, R. J. García López, M. Garczarczyk, D. Garrido, G. Giavitto, N. Godinović, D. Hadasch, D. Häfner, A. Herrero, D. Hildebrand, D. Höhne-Mönch, J. Hose, D. Hrupec, B. Huber,
T. Jogler, S. Klepser, T. Krähenbühl, J. Krause, A. La Barbera, D. Lelas, E. Leonardo, E. Lindfors, S. Lombardi, M. López, E. Lorenz, M. Makariev, G. Maneva, N. Mankuzhiyil, K. Mannheim, L. Maraschi, M. Mariotti, M. Martínez, D. Mazin, M. Meucci, J. M. Miranda, R. Mirzoyan, H. Miyamoto, J. Moldón, A. Moralejo, P. Munar-Androver, D. Nieto, K. Nilsson, R. Orito, I. Oya, S. Paiano, D. Paneque, R. Paoletti, S. Pardo, J. M. Paredes, S. Partini, M. Pasanen, F. Pauss, M. A. Perez-Torres, M. Persic, L. Peruzzo, M. Pilia, J. Pochon, F. Prada, P. G. Prada Moroni, E. Prandini, I. Puljak, I. Reichardt,

R. Reinthal, W. Rhode, M. Ribó, J. Rico, S. Rügamer, A. Saggion, K. Saito, T. Y. Saito, M. Salvati,

K. Satalecka, V. Scalzotto, V. Scapin, C. Schultz,

T. Schweizer, M. Shayduk, S. N. Shore, A. Sillanpää, J. Sitarek, D. Sobczynska, F. Spanier, S. Spiro,

A. Stamerra, B. Steinke, J. Storz, N. Strah, T. Surić,

L. Takalo, H. Takami, F. Tavecchio, P. Temnikov,

T. Terzić, D. Tescaro, M. Teshima, M. Thom,

O. Tibolla, D. F. Torres, A. Treves, H. Vankov,

P. Vogler, R. M. Wagner, Q. Weitzel, V. Zabalza,

F. Zandanel, R. Zanin, M. Fornasa, R. Essig, N. Sehgal, and L. E. Strigari, Searches for dark matter annihilation signatures in the Segue 1 satellite galaxy with the MAGIC-I telescope, JCAP 6 (June, 2011) 35, [\{arXiv: 1103.0477\}].

[27] P. Scott, J. Conrad, J. Edsjö, L. Bergström, C. Farnier, and Y. Akrami, Direct constraints on minimal supersymmetry from Fermi-LAT observations of the dwarf galaxy Segue 1, JCAP 1 (Jan., 2010) 31, [\{arXiv:0909.3300\}].

[28] A. Frebel, J. D. Simon, and E. N. Kirby, Segue 1: An Unevolved Fossil Galaxy from the Early Universe, Astrophys. J. 786 (May, 2014) 74, [\{arXiv:1403.6116\}].

[29] T. J. Cox and A. Loeb, The collision between the Milky Way and Andromeda, MNRAS 386 (May, 2008) 461-474, [\{arXiv:0705.1170\}].

[30] K. Ahn and E. Komatsu, Cosmological lower bound on dark matter masses from the soft gamma-ray background, Phys. Rev. D 71 (Jan., 2005) 021303, [ astro-ph/0412630\}]

[31] A. Klypin, F. Prada, G. Yepes, S. Hess, and S. Gottlober, Halo Abundance Matching: accuracy and conditions for numerical convergence, ArXiv e-prints (Oct., 2013) [\{arXiv:1310.3740\}].

[32] We do not consider the situation when the dark matter particle is identical to its antiparticle. The multiplier should be 1 instead of $\frac{1}{2}$ in this case.

[33] Strictly speaking, $v_{\perp}$ depends on radius. We may roughly estimate it in the following way: since the DM halo is more or less spherically symmetric, $v_{\perp}^{2} \sim v^{2} / 3$, and we may assume that $v$ is approximately equal to the circular orbital speed $v_{\text {orb }}$. Generally speaking, $v_{\text {orb }}$ also depends on radius (to be more precise, on the mass inside the radius, which is not well known for $r \sim 50 \mathrm{kpc}$ ). However, if the density profile can be approximated as $\rho \propto r^{-2}, v_{\text {orb }}=$ const. Since our calculation is estimative, we assume for simplicity that $v_{\perp}$ is constant and $v_{\perp}^{2}=v_{\text {orb }}^{2}\left(r_{\odot}\right) / 3 \simeq 220^{2} / 3 \simeq 10^{4}(\mathrm{~km} / \mathrm{s})^{2}$. 